\title{
Measuring the entropy from shifted boundary conditions
}

\author{
Leonardo Giusti* \\ Dipartimento di Fisica, Università di Milano-Bicocca, \\ and INFN, sezione di Milano-Bicocca \\ Edificio U2, Piazza della Scienza 3 \\ 20126 Milano, Italy. \\ E-mail: Leonardo.Giusti@mib. infn. it
}

\section{Michele Pepe}

INFN, Sezione di Milano-Bicocca

Edificio U2, Piazza della Scienza 3

20126 Milano, Italy.

E-mail: Michele.Pepe@mib.infn.it

We explore a new computational strategy for determining the equation of state of the SU(3) YangMills theory. By imposing shifted boundary conditions, the entropy density is computed from the vacuum expectation value of the off-diagonal components $T_{0 k}$ of the energy-momentum tensor. A step-scaling function is introduced to span a wide range in temperature values. We present preliminary numerical results for the entropy density and its step-scaling function obtained at eight temperature values in the range $T_{c}-15 T_{c}$. At each temperature, discretization effects are removed by simulating the theory at several lattice spacings and by extrapolating the results to the continuum limit. Finite-size effects are always kept below the statistical errors. The absence of ultraviolet power divergences and the remarkably small discretization effects allow for a precise determination of the step-scaling function in the explored temperature range. These findings establish this strategy as a viable solution for an accurate determination of the equation of state in a wide range of temperature values.

31st International Symposium on Lattice Field Theory LATTICE 2013

July 29th - August 3rd, 2013

Mainz, Germany

\footnotetext{
*Speaker.
} 


\section{Introduction}

The standard approach to determine the Equation of State of a gauge theory on the lattice requires the numerical computation of the free energy density [1]. This quantity is usually measured by computing its derivative with respect to the bare coupling constant via Monte Carlo simulations, and then integrating it back analytically. The quartic ultraviolet divergent term is removed by subtracting the very same quantity at zero temperature. Apart from the expansion in the bare coupling constant, this strategy requires to accommodate two very different scales at the same lattice spacing: the temperature $T$ and the smallest inverse correlation length of the zero temperature theory. Only this way discretization and finite volume effects can be kept under control. Although this approach has shown to be successful at low temperature, the zero-temperature simulations needed for the subtraction at large temperatures become quickly very expensive. This problem prevented numerical computations to access temperatures larger than a few $T_{c}$. The situation improved with the generalization of the method via the half-temperature subtraction [2]. However, the expansion in the bare parameters is still needed and the simulations remain rather demanding.

In this contribution we present a new computational strategy to avoid the two-scale problem and, at the same time, the expansion in the bare coupling. The entropy density is extracted from the expectation value of a local operator computed at the desired temperature, and no ultraviolet power subtractions are needed.

The basic new ingredient is the use of shifted boundary conditions in the temporal direction [3, $4,5]$. In this set up the entropy density can be determined from the expectation value of the off-diagonal components $T_{0 k}$ of the energy-momentum tensor [5], a quantity which renormalizes multiplicatively in the SU(3) gauge theory on the lattice. Finite volume effects are exponentially small in $(M L)$, where $M$ is the lightest screening mass of the theory and $L$ is the linear size in the spatial directions. At large $T$, the temperature is the relevant scale in the system: the dominant discretization effects are proportional to $(a T)^{2}$, and $M$ is proportional to $T$ (more precisely to $(g T)$ or $\left(g^{2} T\right)$ depending on the temperature). Since the observable is local, the computational effort is volume independent at fixed statistical errors. One can thus simulate large spatial volumes to account for the small prefactor which enters the expression of the screening mass, and still keep discretization effects under control. We test here these ideas in the SU(3) Yang-Mills theory. They can, however, be easily generalized to gauge theories with fermions like QCD.

\section{Entropy density from shifted boundary conditions}

The SU(3) Yang-Mills theory at finite temperature can be formulated in the Euclidean path integral formalism by imposing on the fields periodic boundary conditions in the compact direction up to a shift $\boldsymbol{\xi}$ in the spatial direction $[3,4,5]$

$$
A_{\mu}\left(L_{0}, \boldsymbol{x}\right)=A_{\mu}\left(0, \boldsymbol{x}-L_{0} \boldsymbol{\xi}\right),
$$

where $A_{\mu}$ is the gauge field and $L_{0}$ is the time extension. In the thermodynamic limit, the invariance of the dynamics under the $\mathrm{SO}(4)$ group implies that the free energy density $f\left(L_{0}, \boldsymbol{\xi}\right)$ satisfies

$$
f\left(L_{0}, \boldsymbol{\xi}\right)=f\left(L_{0} \sqrt{1+\boldsymbol{\xi}^{2}}, \mathbf{0}\right) .
$$


Thus the free energy depends on the length of the compact direction $\beta=L_{0} \sqrt{1+\xi^{2}}=T^{-1}$ which fixes the inverse temperature of the system, while it is independent on its orientation with respect to the space directions. This redundancy implies that the total energy and momentum distributions of the thermal theory are related, and interesting Ward identities (WIs) follow. In particular, one obtains that

$$
\left\langle T_{0 k}\right\rangle_{\boldsymbol{\xi}}=\frac{\xi_{k}}{1-\xi_{k}^{2}}\left[\left\langle T_{00}\right\rangle_{\boldsymbol{\xi}}-\left\langle T_{k k}\right\rangle_{\boldsymbol{\xi}}\right]
$$

where $\langle\cdot\rangle_{\boldsymbol{\xi}}$ stands for the vacuum expectation value computed in the thermal quantum system with shift $\boldsymbol{\xi}$, and $T_{\mu \nu}$ is the energy-momentum tensor of the theory. For a non-vanishing shift, it then follows that the entropy density $s(T)$ is given by

$$
\frac{s(T)}{T^{3}}=-\frac{L_{0}^{4}\left(1+\xi^{2}\right)^{3}}{\xi_{k}}\left\langle T_{0 k}\right\rangle_{\boldsymbol{\xi}}, \quad T=\frac{1}{L_{0} \sqrt{1+\boldsymbol{\xi}^{2}}}
$$

Remarkably, the entropy density can be obtained directly from the vacuum expectation value of the off-diagonal component $T_{0 k}$ of the energy-momentum tensor which does not vanish since the shift softly breaks the parity symmetry. Based on the Eq. (2.4), a step-scaling function $\Sigma(T, r)$ for the normalized entropy density can be defined as

$$
\Sigma(T, r)=\frac{s\left(T^{\prime}\right) / T^{\prime 3}}{s(T) / T^{3}}=\frac{\left(1+\boldsymbol{\xi}^{\prime 2}\right)^{3} \xi_{k}}{\left(1+\xi^{2}\right)^{3} \xi_{k}^{\prime}} \frac{\left\langle T_{0 k}\right\rangle_{\xi^{\prime}}}{\left\langle T_{0 k}\right\rangle_{\boldsymbol{\xi}}},
$$

where $\boldsymbol{\xi}$ and $\boldsymbol{\xi}^{\prime}$ are two different shifts. Since $L_{0}$ is held fixed, the step $r$ in the temperature is given by the ratio $r=T^{\prime} / T=\sqrt{1+\xi^{2}} / \sqrt{1+\xi^{\prime 2}}$. Following the approach in Ref. [7], the entropy density at a given temperature can then be obtained by solving the recursion relation

$$
v_{0}=\frac{s\left(T_{0}\right)}{T_{0}^{3}}, \quad v_{k+1}=\Sigma_{s}\left(T_{k}, r\right) v_{k}, \quad T_{k}=T_{0} r^{k},
$$

once the entropy density $v_{0}$ is computed at temperature $T_{0}$.

\section{Entropy density on the lattice}

We set up the SU(3) Yang-Mills theory on a four-dimensional lattice of size $L_{0} \times L^{3}$ and spacing $a$ by discretizing the gluons with the standard Wilson plaquette action. We impose periodic boundary conditions in the spatial directions and shifted boundary conditions along the compact direction

$$
U_{\mu}\left(L_{0}, \boldsymbol{x}\right)=U_{\mu}\left(0, \boldsymbol{x}-L_{0} \boldsymbol{\xi}\right),
$$

where $\boldsymbol{\xi}$ is the shift vector and $U_{\mu}(x)$ are the gauge links. We consider the clover formulation of the energy-momentum tensor on the lattice [6]

$$
T_{\mu v}=\frac{\beta}{6}\left\{F_{\mu \alpha}^{a} F_{v \alpha}^{a}-\frac{1}{4} \delta_{\mu \nu} F_{\alpha \beta}^{a} F_{\alpha \beta}^{a}\right\}
$$


where $\beta=6 / g_{0}^{2}$, and $g_{0}$ is the bare coupling constant. The field strength tensor is defined as

$$
F_{\mu \nu}^{a}(x)=-\frac{i}{4 a^{2}} \operatorname{Tr}\left\{\left[Q_{\mu \nu}(x)-Q_{\nu \mu}(x)\right] T^{a}\right\},
$$

where

$$
\begin{aligned}
Q_{\mu v}(x) & =U_{\mu}(x) U_{v}(x+a \hat{\mu}) U_{\mu}^{\dagger}(x+a \hat{v}) U_{v}^{\dagger}(x) \\
& +U_{v}(x) U_{\mu}^{\dagger}(x-a \hat{\mu}+a \hat{v}) U_{v}^{\dagger}(x-a \hat{\mu}) U_{\mu}(x-a \hat{\mu}) \\
& +U_{\mu}^{\dagger}(x-a \hat{\mu}) U_{v}^{\dagger}(x-a \hat{\mu}-a \hat{v}) U_{\mu}(x-a \hat{\mu}-a \hat{v}) U_{v}(x-a \hat{v}) \\
& +U_{v}^{\dagger}(x-a \hat{v}) U_{\mu}(x-a \hat{v}) U_{v}(x+a \hat{\mu}-a \hat{v}) U_{\mu}^{\dagger}(x) .
\end{aligned}
$$

On the lattice translational invariance is broken down to a discrete sub-group, and the energymomentum tensor has to be renormalized. The momentum density renormalizes multiplicatively $T_{0 k}^{\mathrm{R}}=Z_{T} T_{0 k}$, and its renormalization constant $Z_{T}$ is fixed by imposing suitable WIs $[4,5]$. As a consequence $Z_{T}$ depends only on the bare coupling constant and, up to discretization effects, it is independent on the volume, the temperature, the shift parameter, etc. Ultimately which WIs and/or kinematics yield the most accurate result must be investigated numerically. The factor $Z_{T}$ cancels out in the lattice definition of the stepscaling function

$$
\Sigma(T, r)=\frac{\left(1+\boldsymbol{\xi}^{\prime 2}\right)^{3} \xi_{k}}{\left(1+\boldsymbol{\xi}^{2}\right)^{3} \xi_{k}^{\prime}} \frac{\left\langle T_{0 k}\right\rangle_{\boldsymbol{\xi}^{\prime}}}{\left\langle T_{0 k}\right\rangle_{\boldsymbol{\xi}}}
$$

which has a universal continuum limit as it stands. In the remaining part of these proceedings we will focus on the numerical determination of the stepscaling function.

\section{Numerical computation}

We have simulated the SU(3) Yang-Mills theory by sweeping the lattice with 1 heatbath and 3 over-relaxation updates of all link variables. At each value of $\beta$ and $L_{0} / a$, we have measured $\left\langle T_{0 k}\right\rangle_{\boldsymbol{\xi}}$ for two values of the shift, $\boldsymbol{\xi}^{\prime}=(1,0,0)$ and $\boldsymbol{\xi}=(1,1,1)$, corresponding to a step of $r=$ $\sqrt{2}$ in the temperature. In order to extrapolate $\Sigma(T, \sqrt{2})$ to the continuum limit, at each temperature

\begin{tabular}{ccccc}
\hline$T / T_{0}$ & $L / a$ & $L_{0} / a$ & $\beta$ & $T L$ \\
\hline $1 / \sqrt{2}$ & 80 & 3 & 6.0403 & 13.4 \\
$1 / \sqrt{2}$ & 96 & 3 & 6.0403 & 22.6 \\
$1 / \sqrt{2}$ & 96 & 4 & 6.2257 & 12.0 \\
$1 / \sqrt{2}$ & 128 & 5 & 6.3875 & 12.8 \\
\hline 1 & 80 & 3 & 6.2670 & 13.4 \\
1 & 96 & 3 & 6.2670 & 22.6 \\
1 & 96 & 4 & 6.4822 & 12.0 \\
1 & 128 & 5 & 6.6575 & 12.8 \\
\hline$\sqrt{2}$ & 80 & 3 & 6.5282 & 13.4 \\
$\sqrt{2}$ & 96 & 3 & 6.5282 & 22.6 \\
$\sqrt{2}$ & 96 & 4 & 6.7533 & 12.0 \\
$\sqrt{2}$ & 128 & 5 & 6.9183 & 12.8 \\
\hline 2 & 80 & 3 & 6.7791 & 13.4 \\
2 & 96 & 3 & 6.7791 & 22.6 \\
2 & 96 & 4 & 7.0201 & 12.0 \\
2 & 128 & 5 & 7.2068 & 12.8 \\
\hline $2 \sqrt{2}$ & 80 & 3 & 7.0694 & 13.4 \\
$2 \sqrt{2}$ & 96 & 3 & 7.0694 & 22.6 \\
$2 \sqrt{2}$ & 96 & 4 & 7.3100 & 12.0 \\
$2 \sqrt{2}$ & 128 & 5 & 7.4963 & 12.8 \\
\hline 4 & 80 & 3 & 7.4120 & 13.4 \\
4 & 96 & 3 & 7.4120 & 22.6 \\
4 & 96 & 4 & 7.6541 & 12.0 \\
4 & 128 & 5 & 7.8435 & 12.8 \\
\hline $4 \sqrt{2}$ & 80 & 3 & 7.7039 & 13.4 \\
$4 \sqrt{2}$ & 96 & 3 & 7.7039 & 22.6 \\
$4 \sqrt{2}$ & 96 & 4 & 7.9489 & 12.0 \\
$4 \sqrt{2}$ & 128 & 5 & 8.1405 & 12.8
\end{tabular}

Table 1: The parameters used in the numerical study. For each parameter set, simulations with two shifts $\boldsymbol{\xi}^{\prime}=(1,0,0)$ and $\boldsymbol{\xi}=(1,1,1)$ have been carried out. This corresponds to the two temperatures: $T=\left(2 L_{0}\right)^{-1}$ and $T^{\prime}=\left(\sqrt{2} L_{0}\right)^{-1}$. 


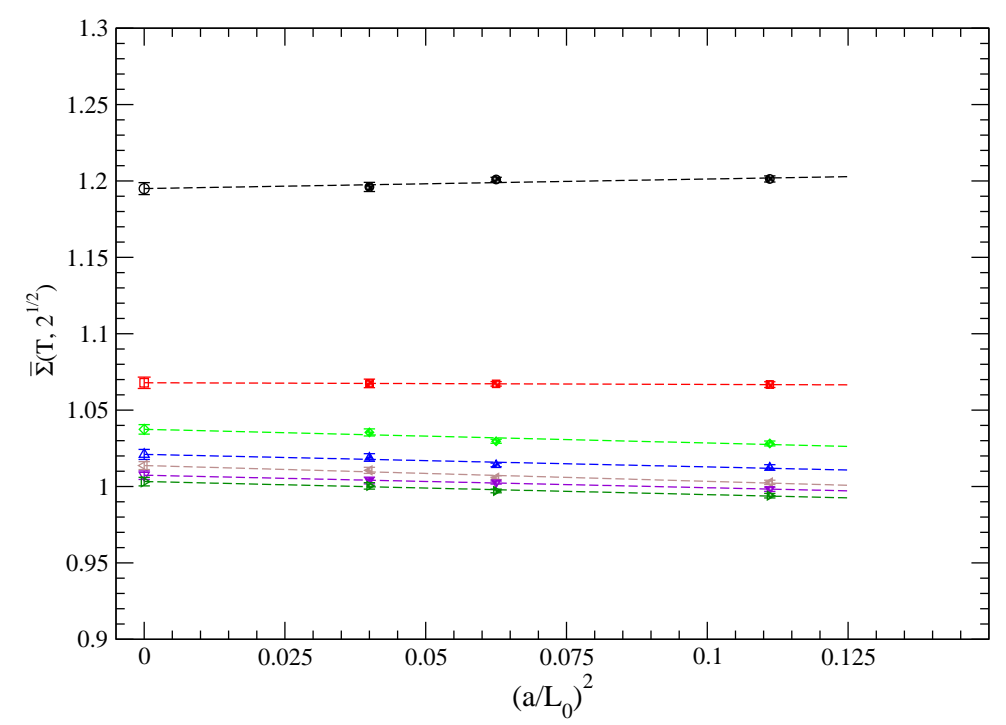

Figure 1: Continuum limit extrapolation of the entropy step-scaling function $\bar{\Sigma}(T, \sqrt{2})$ at the 7 temperatures listed in Table 1. The temperature increases from top to bottom. The dashed lines correspond to linear fits of the numerical data.

we have collected data at three different values of the lattice spacing $L_{0} / a=3$, 4 , and 5 ; runs at $L_{0} / a=6$ are currently in progress to have a better control on the systematics due to the extrapolation. We have measured $\Sigma(T, \sqrt{2})$ at 7 temperatures in the range $T_{0} / \sqrt{2}-4 \sqrt{2} T_{0}$, with the values separated by steps of about $\sqrt{2}$ to match the value of $r$. Our reference temperature has been fixed to $T_{0}=L_{\max }^{-1}$, where $L_{\max }$ is taken from Ref. [8]. It corresponds to $T_{0} \simeq 1.802 T_{c}$, where $T_{c}$ is the critical temperature computed in Ref. [9]. In the first three steps $(k=-1,0,1)$ the value of $\beta$ of each run has been fixed from $r_{0} / a$, by requiring that $L_{\max } / r_{0}=0.738(16)$ [11]. For each pair of steps $k=2 j, 2 j+1$, with $j \geq 1$, we interpolate quadratically in $\ln (L / a)$ each set of data at constant $\bar{g}^{2}\left(L_{j}\right)$ in Table A.1 of Ref. [7] supplemented by the corresponding data in Table A.4

$$
\beta=a_{j}+b_{j} \ln \left(\frac{L}{a}\right)+c_{j} \ln ^{2}\left(\frac{L}{a}\right),
$$

and we fix $\beta$ by requiring that $T_{2 j} a=a / L_{j}$ and $T_{2 j+1} a=\sqrt{2} a / L_{j}$. The $\beta$ values obtained are reported in Table 1, together with the spatial and time extent of the lattices simulated. To keep finite volume effects below the statistical errors, we have chosen $T L \geq 12$. At each coarser lattice spacing, we have also simulated a smaller volume to verify that finite volume effects are below the statistical errors. Having large lattices does not increase the cost, since the latter is volume independent thanks to the locality of the observable.

\section{Results and conclusions}

The step-scaling function in the free theory, $\Sigma_{0}(T, \sqrt{2})$, can be computed analytically on the lattice. It has small discretization effects of the order of a few $\%$ in the range of $L_{0} / a$ we are 


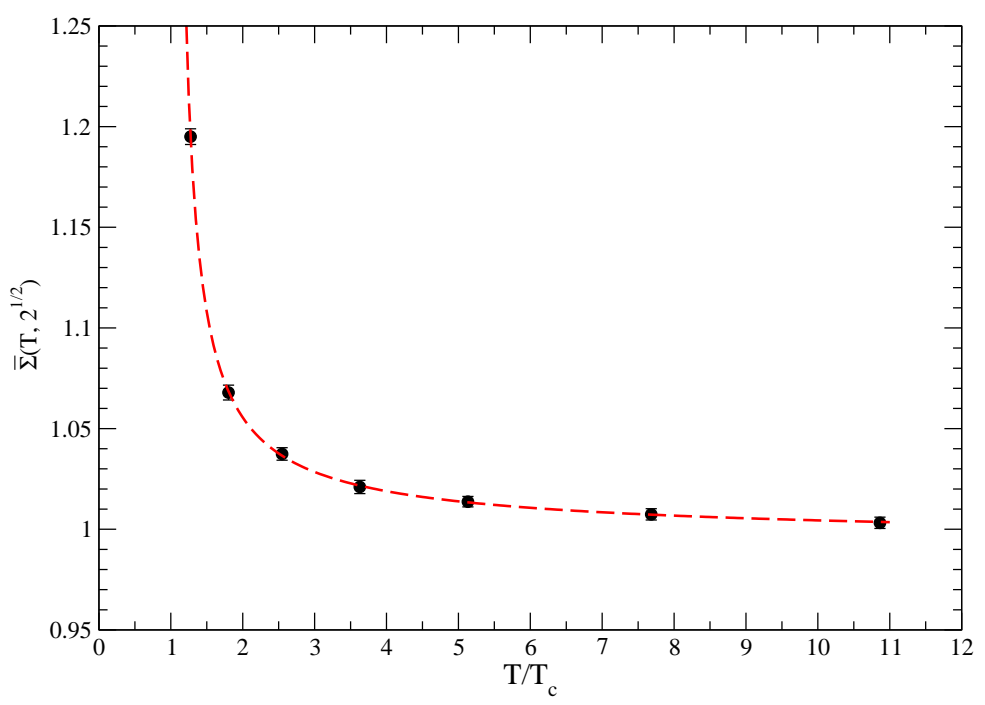

Figure 2: The temperature dependence of the entropy step-scaling function in the continuum limit. Error bars take into account statistical errors only. The dashed line is an interpolation of the points to guide the eyes.

interested in [5]. This fact is confirmed in the interacting theory by our preliminary results shown in Fig. 1, where the difference

$$
\bar{\Sigma}(T, \sqrt{2})=\Sigma(T, \sqrt{2})-\left(\Sigma_{0}\left(L_{0}\right)-1\right)
$$

is plotted as a function of $\left(a / L_{0}\right)^{2}$. The residual discretization effects in $\bar{\Sigma}(T, \sqrt{2})$ are at the permille level already at $L_{0} / a=3$. The linear extrapolation in $\left(a / L_{0}\right)^{2}$ provides a satisfactory fit of the numerical data, as shown by the dashed lines in the plot. When the data at $L_{0} / a=6$ will become available, we will be able to quantify also the systematics due to the continuum limit extrapolation. At that point it may be useful to attempt a global fit of all data points by parameterizing the coefficient of the discretization effects. In Fig. 2 we plot the continuum limit values of the step-scaling function versus the temperature, where the errors are statistical only. The temperatures at which the step-scaling function has been measured are not always precisely related by a factor $\sqrt{2}$. However, by interpolating the results of the step-scaling function, the step $\sqrt{2}$ in the temperature can be accurately enforced and the temperature dependence of $s(T) / T^{3}$ can be reconstructed. For the sake of the presentation, in the Fig. 3 we show the temperature dependence of $s(T) / T^{3}$ obtained from the entropy step-scaling function by fixing the overall normalization from the results for the entropy density at $T=4.1 T_{c}$ published in [3]. The statistical uncertainty on that measurement dominates the error bars. We expect to improve significantly the accuracy of these results once $Z_{T}$ will be fixed from the WIs. A first step in this direction has already been taken at this conference [12].

Our preliminary results establish the strategy followed in this work as a viable and efficient solution for determining accurately the equation of state in a wide range of temperature values. The cost of these simulations is moderate, as proved by the fact that all computations presented here 


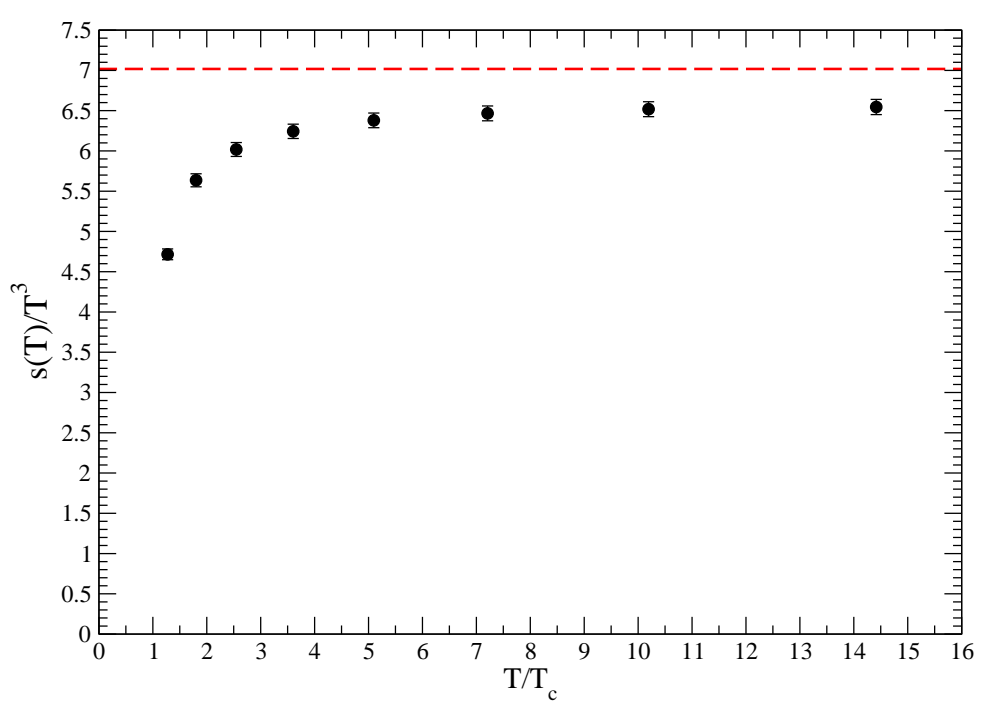

Figure 3: The normalized entropy density as a function of $T / T_{c}$. The dashed line is the Stefan-Boltzmann result $s_{\mathrm{SB}} / T^{3}=32 \pi^{2} / 45$.

have been done using a few millions of core hours on a BG/Q. Simulations to properly quantify the systematic errors due to the continuum limit extrapolation are underway.

We thank H. B. Meyer and D. Robaina for discussions. Simulations have been performed on Blue Gene/Q at CINECA (CINECA-INFN agreement) and on the Turing machine at the University of Milano-Bicocca. We thank these Institutions for the generous allocation, the support and the technical help. This work was partially supported by the MIUR-PRIN contract 20093BMNNPR and by the INFN SUMA project.

\section{References}

[1] G. Boyd et al., Nucl. Phys. B 469 (1996) 419.

[2] S. .Borsanyi, G. Endrodi, Z. Fodor, S. D. Katz and K. K. Szabo, JHEP 1207, 056 (2012).

[3] L. Giusti and H. B. Meyer, Phys. Rev. Lett. 106, 131601 (2011).

[4] L. Giusti and H. B. Meyer, JHEP 1111, 087 (2011).

[5] L. Giusti and H. B. Meyer, JHEP 1301, 140 (2013).

[6] S. Caracciolo, G. Curci, P. Menotti and A. Pelissetto, Annals Phys. 197, 119 (1990).

[7] M. Luscher, R. Sommer, P. Weisz and U. Wolff, Nucl. Phys. B 413 (1994) 481 [hep-lat/9309005].

[8] S. Capitani et al. [ALPHA Collaboration], Nucl. Phys. B 544, 669 (1999).

[9] B. Lucini, M. Teper and U. Wenger, JHEP 0401 (2004) 061 [hep-lat/0307017].

[10] R. Sommer, Nucl. Phys. B 411, 839 (1994).

[11] S. Necco and R. Sommer, Nucl. Phys. B 622, 328 (2002).

[12] D. Robaina and H. B. Meyer, PoS (LATTICE 2013) 323 [arXiv:1310.6075] . 